OPEN ACCESS

Edited by:

Francesca Romana Ponziani, Fondazione Policlinico Universitario

Agostino Gemelli IRCCS, Italy

Reviewed by:

Sara Montagnese,

University of Padua, Italy

Piero Amodio,

University of Padua, Italy

*Correspondence:

Bimei Jiang

jiangbimei@163.com

${ }^{t}$ These authors have contributed equally to this work

Specialty section:

This article was submitted to Microbiome in Health and Disease,

a section of the journal

Frontiers in Cellular and

Infection Microbiology

Received: 18 August 2020

Accepted: 08 January 2021

Published: 22 February 2021

Citation:

Liu J, Xu Y and Jiang B (2021) Novel Insights Into Pathogenesis and

Therapeutic Strategies of Hepatic Encephalopathy, From the Gut Microbiota Perspective.

Front. Cell. Infect. Microbiol. 11:586427.

do: 10.3389/fcimb.2021.586427

\section{Novel Insights Into Pathogenesis and Therapeutic Strategies of Hepatic Encephalopathy, From the Gut Microbiota Perspective}

\author{
Jiachen Liu ${ }^{1+}$, Yantao $\mathrm{Xu}^{1+}$ and Bimei Jiang ${ }^{2 *}$ \\ ${ }^{1}$ Xiangya Medical College of Central South University, Changsha, China, ${ }^{2}$ Department of Pathophysiology, Sepsis Translational \\ Medicine Key Laboratory of Hunan Province, Xiangya School of Medicine, Central South University, Changsha, China
}

Since the 1950s, gradual changes in the gut microbiota of patients with hepatic encephalopathy have been observed. Previous research has indicated potential associations between the gut and brain, and the gut microbiota is becoming a hot topic in research on diseases of the nervous system. However, for the past few decades, studies of hepatic encephalopathy have been restricted to controlling the gut microbiota during macroscopic manipulation, such as probiotic intervention, while its clinical use remains controversial, and the cellular mechanisms underlying this condition are still poorly understood. This thesis seeks to comprehensively understand and explain the role of gut microbiota in hepatic encephalopathy as well as analyze the effects of intervention by regulating the gut microbiota. Evidence is presented that shows that dysbiosis of the gut microbiota is the primary pathological driver of hepatic encephalopathy and impacts pathologic progression via complex regulatory networks. As a result, suggestions were identified for future mechanistic research and improvements in therapeutic strategies for hepatic encephalopathy.

Keywords: gut microbiota, hepatic encephalopathy, gut-liver-brain axis, probiotic, fecal microbiota transplantation, antibiotics

\section{HIGHLIGHTS}

Pathogenesis of hepatic encephalopathy are summarized from gut microbiota perspective.

- Intervention effects of hepatic encephalopathy via regulating the gut microbiota are analyzed.

- Novel perspectives on therapeutic strategy of hepatic encephalopathy are introduced. 


\section{INTRODUCTION}

Hepatic encephalopathy (HE) is one of the most severe complications of cirrhosis and involves alteration of consciousness, which is directly related to liver failure (Bajaj, 2018). According to the latest guidelines published in 2018, HE is defined as brain dysfunction caused by liver failure and/or portal-systemic blood shunting that produces a spectrum of neurological/psychiatric abnormalities ranging from subclinical alterations to coma (Montagnese et al., 2019). HE is still indisputably associated with hepatic insufficiency; therefore, it is reasonable to characterize this encephalopathy as HE (Yanny et al., 2019). According to epidemiological investigations, the prevalence of HE is approximately 30 to $45 \%$, which is accompanied by an increase in mortality (Patidar and Bajaj, 2015; Xu et al., 2019). In the United States, the economic burden of hospitalization for HE amounts to more than $\$ 11.9$ billion each year (Hirode et al., 2019), which causes significant increases in patient mobility, morbidity, and care utilization (Jang et al., 2020).

The number of microorganisms inhabiting the gut (the largest surface area in the body) has been estimated to exceed 100 quintillion (Biver et al., 2019). In the local immune system, the gut epithelium is a major line of defense in which epithelial cells provide a physical barrier and work in concert with immune and stromal cells to fight off microbes and toxins. Increasing evidence suggests that gut barrier dysfunction can lead to liver failure, which can in turn aggravate intestinal mucosal injury (Arab et al., 2018; Xu et al., 2018) (Figure 1). During chronic liver failure, ammonia accumulation in the brain leads to Alzheimer type 2 astrocytosis, which has been highlighted as neuropathological characteristics of HE (Butterworth, 2019). Besides, maintained inflammation caused by hyperammonemias will promote the development of systemic and central inflammation (Aitbaev et al., 2017), which will cause activation of the microglia indicative of neuroinflammation (Rodrigo et al., 2010). Therefore, there is a potential but important interaction between the gut microbiota and HE, which will provide a novel perspective on $\mathrm{HE}$ and inform the development of future interventions (Bajaj, 2019).

\section{HE AND MICROBIOTA: INTERACTION}

The key to understanding the pathogenesis of $\mathrm{HE}$ is to adequately describe the physiological fluctuations and pathological shifts of the gut-brain-liver axis as well as gut microbiota metabolic interactions. A more detailed account of the pathological process is given in the following section.

\footnotetext{
Abbreviation: HE, hepatic encephalopathy; SCFA, short-chain fatty acids; FMT, fecal microbiota transplantation; MRI, magnetic resonance imaging; MRS, magnetic resonance spectroscopy; DTI, diffusion tensor imaging; TNF, tumor necrosis factor; LBP, lipopolysaccharide binding protein; TLR, toll-like receptor; IL, interleukin.
}

\section{The Role of the Gut-Brain-Liver Axis in the Pathogenesis of HE}

The gut-liver-brain axis refers to the bidirectional relationship between the gut and its microbiota, the liver, and the brain, resulting from the integration of signals generated by dietary, genetic, and environmental factors (Wiest et al., 2017; Mancini et al., 2018). A healthy, homeostatic condition of the gut-liverbrain axis provides two shields for the brain against the hazardous contents in the gut: one is the homeostasis of gut permeability, and the other is normal hepatic function (Mancini et al., 2018) (Figure 2). The gut is open to the external environment, resulting in a convenient pathway for microbiota colonization, and can be easily affected by changes in the environment. In addition, the gut is responsible for digestion and absorption, indicating the need for a strong permeability function to prevent any toxins in the gut from reaching the capillaries. However, the liver is a recipient and filter of nutrients, bacterial products, toxins and metabolites, both endogenous an exogenous (Wiest et al., 2017). The collapse of either shield causes several mutually reinforced abnormal states and leads to diseases such as cirrhosis, portal hypertension, and HE.

As a late complication of cirrhosis, $\mathrm{HE}$ is considered to be a typical disease owing to abnormal gut-liver-brain function, but the understanding of its pathogenesis is poor. Increasing evidence shows that alteration in the structure of the gut microbiota and its metabolic by-products, as well as a background of local and systemic inflammation and leaky gut, drive the development of HE (Azhari and Swain, 2018; Manzhalii et al., 2019; Jaffe et al., 2020).

The relationship between the gut-liver-brain axis and liver disease are likely to be described as a "chicken or egg" problem (Arab et al., 2018). The collapse of functions of the gut barrier is due to various causes, such as local infection and bacterial translocation, which can alter gut permeability, bacterial translocation, and systemic and local inflammation; modify the microbiota composition; and reduce commensal bacteria and their metabolites, such as short-chain fatty acids (SCFAs) (Aguirre Valadez et al., 2016; Fukui and Wiest, 2016). This phenomenon provides a condition for enteric endotoxins and harmful metabolites to enter the blood through the gut mucosa, thus damaging liver and brain functions as well as causing HE, which might be the initiating factor (Aguirre Valadez et al., 2016; Fukui and Wiest, 2016) (Figure 3).

However, liver function also affects the barrier function of the gut. Anatomically, the liver and gut are connected by the portal vein system (Covey et al., 2004). The normal functioning of the portal system as well as normal liver function allow harmful metabolic substances produced in the gut to be decomposed in the liver, thus maintaining the remaining part of the blood with normal levels of toxins (Wiest et al., 2017). Regulation of ammonia levels entering the portal circulation depends on the urea cycle in the liver as well as on muscle and kidney metabolism (Milosevic et al., 2019; Raj et al., 2020). Unfortunately, the loss of normal liver function and abnormal conditions, such as portal-systemic syndrome during the disease, reduce metabolic function. In cirrhosis, the increase of plasma 

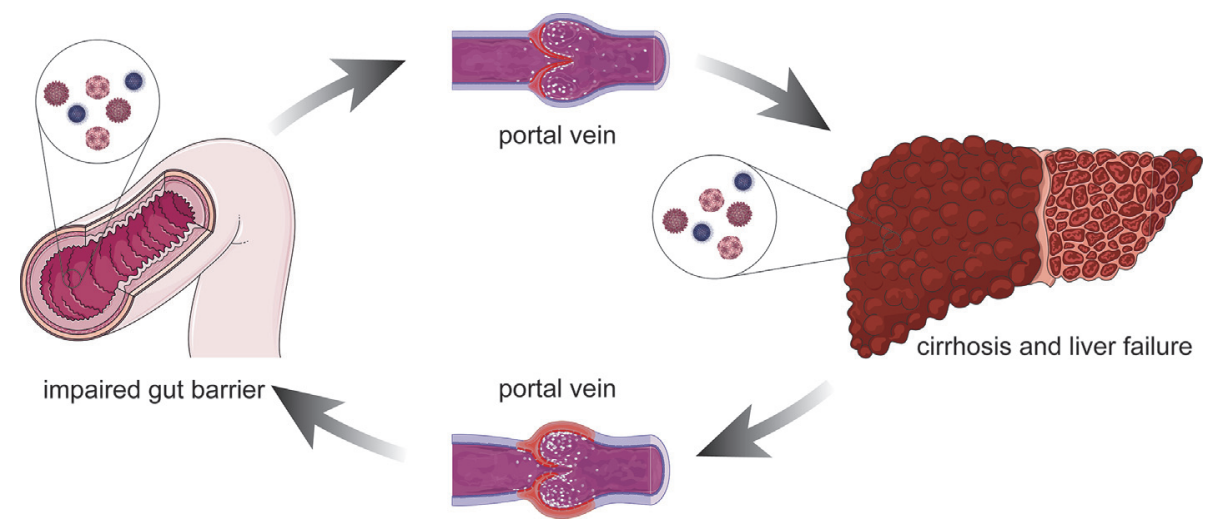

FIGURE 1 | Association between the damaged intestinal mucosa liver failure. Altered immune function such as impaired gut barrier, bacterial products can reach the liver through the portal vein and might lead to a pro-inflammatory response. The pro-inflammatory stimulus facilitates hepatocyte damage and central process that promotes liver inflammation. On the other hand, the defunctionalization of the liver carries on impairing the gut barrier due to the portal hypertension. Left untreated, it can lead to cirrhosis and liver failure.

ammonia in cirrhosis is mainly due to portal-systemic shunting, since ammonia is a substance highly extracted by the liver. Ammonia enters the circulation and then penetrates the blood brain barrier, leading to neuropsychiatric complications such as HE (Milosevic et al., 2019).

\section{The Role of Gut Microbiota in the Pathogenesis of HE From the Gut-Brain- Liver Axis Perspective}

Although the pathogenesis of HE is unclear, the hypothesis that the microbiota plays an important role in this condition has been confirmed. Some evidence shows that the higher risk of microbiota dysbiosis in cirrhotic patients, with the subsequent clinical implications, is principally due to the various pathological interactions between the liver and the gastrointestinal tract. The alteration in gut motility, the higher $\mathrm{pH}$, and the reduced bile acid concentrations in the colons of patients with cirrhosis may lead to a failure to control gut bacterial overgrowth.

In addition, cirrhosis impairs the homeostatic role of the liver in the systemic immune response. Damage to the reticuloendothelial system compromises the liver immune surveillance function exerted by Kupffer cells and sinusoidal endothelial cells, and the reduced hepatic synthesis of proteins involved in innate immunity and pattern recognition hinders the bactericidal ability of phagocytic cells (Katz et al., 1991; Sipeki et al., 2014). Monocyte spreading, chemotaxis, and neutrophil activity are also significantly reduced in patients with cirrhosis compared with controls (Fiuza et al., 2000). This phenomenon can in turn compromise the gut barrier and facilitate bacterial translocation, an increased risk of gut bacterial infections and decompensated liver disease (Schnabl and Brenner, 2014; Betrapally et al., 2017).

The bacterial composition of the sigmoid colon in patients with HE was significantly altered (Bajaj et al., 2012b). Recent studies have demonstrated lower Roseburia and higher
Enterococcus, Veillonella, Megasphaera, and Burkholderia among sigmoid colonic mucosal microbiota in HE groups compared to controls. Moreover, genera including Blautia, Fecalibacterium, Roseburia, and Dorea can be considered biomarkers of cognition and inflammation that are related to acetylcholinesterase inhibitors (Bajaj et al., 2012a; Zhang et al., 2013). In addition, Bajaj and his colleagues demonstrated that Enterobacteriaceae, Fusobacteriaceae, and Veillonellaceae were positively and Ruminococcaceae negatively related to inflammation (Bajaj et al., 2012b) (Table 1). However, these studies are all cross-sectional therefore can hardly provide little evidence for causal presumption, but there is still some value to be considered as some candidate biomarker, indicating that the pathogenesis of HE may be associated with the interaction between the immune system and microbiota (Arab et al., 2018).

In a case report conducted by Kao, a 57-year-old man suffering from grades 1-2 HE with liver cirrhosis treated by FMT (Kao et al., 2016), despite a reduction in the relative abundance of Lachnospiraceae following FMT, showed an obvious improvement in cognition, suggesting that other microbial taxa and metabolic activity are involved. However, the result is from a single case report, so the universality of this conclusion remains in doubt. Interestingly, the inhibitory control test and Stroop test scores reverted to baseline by week 14, suggesting that the beneficial effect of fecal microbiota transplantation (FMT) is transient and that repeated therapy would be required to maintain the response (Kao et al., 2016). The results laterally demonstrate the correlation between gut microbiota and liver damage, indicating that persistent dysfunction of gut microbiota is one of the conditions for the development of $\mathrm{HE}$ and that liver function damage will facilitate this process. Although this study is the first report of treating HE with FMT and is well organized, it still has some unfortunate limitations, such as the lack of data on ammonia levels, as these parameters were not determined at a standardized time or in the 

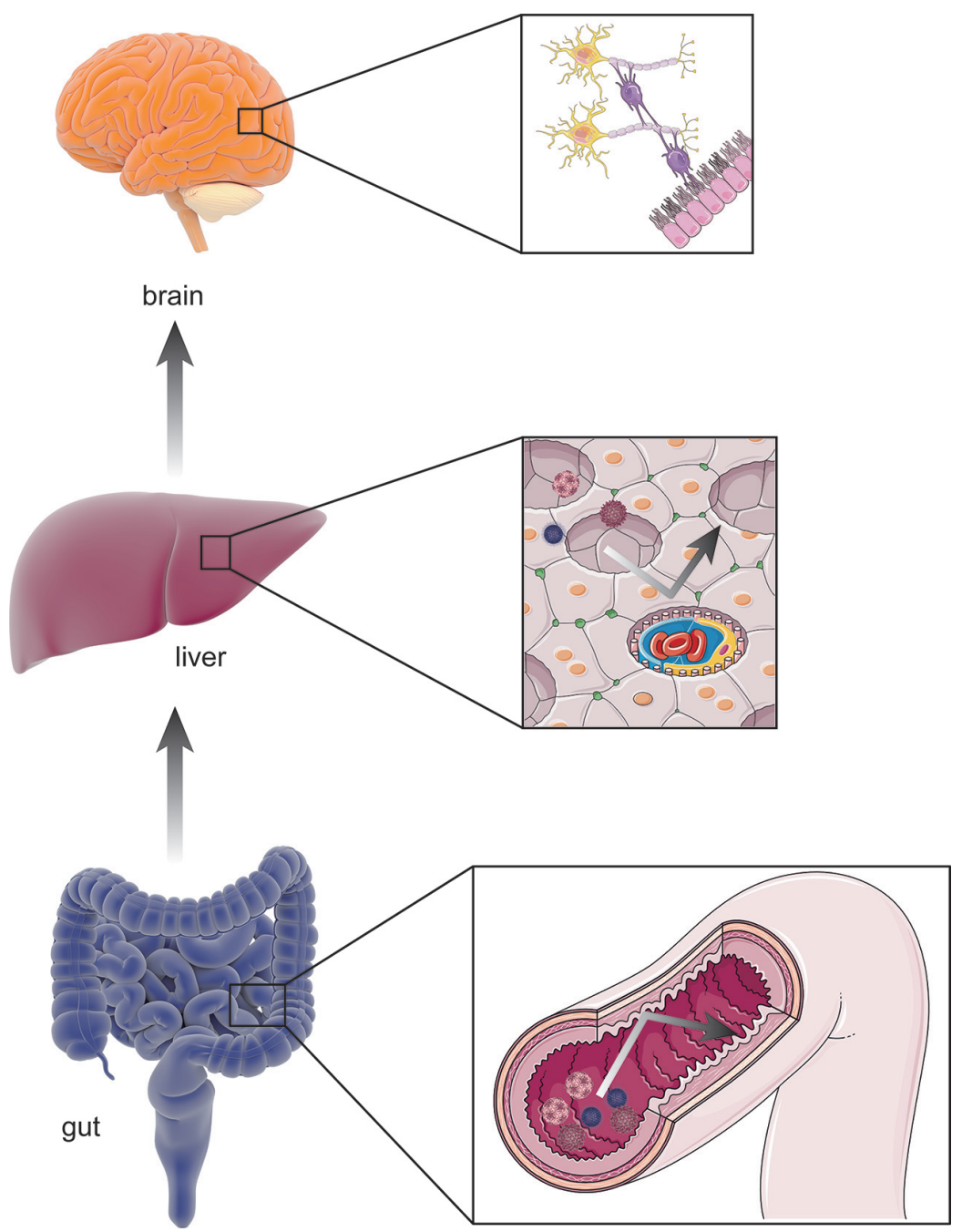

FIGURE 2 | The healthy gut-liver-brain axis. A healthy gut-liver-brain axis contains gut and its microbiota, the liver, and the brain. In the normal condition, two "shields" including homeostasis of intestinal permeability as physical barrier and the normal hepatic function of amino-metabolism prevent potential pathogens and gut enteroendotoxin from hamming other organs (such as brain) through blood stream.

fasting state, which suggests that we can reproduce such studies in animal models for more relevant data.

For changes in neuronal system, some very ancient studies of the end of the fifties of 20th century suggested the effect of oral neomycin on fecal flora so as to enterobatteriacae, such as E. coli and Streptococcus faecalis, with improvement in HE, especially in chronic neuropsychiatric disorder (Dawson et al., 1957; Summerskill, 1958). Ahluwalia conducted a study to define the individual contribution of specific gut bacterial taxa towards astrocytic and neuronal changes in brain function using multimodal magnetic resonance imaging (MRI) in patients with cirrhosis with or without HE (Ahluwalia et al., 2016). The study included 87 subjects with $\mathrm{HE}$ who underwent systemic inflammatory assessment, cognitive testing, stool microbiota analysis, and brain MRI analysis. Magnetic resonance spectroscopy (MRS) showed that the increased glutamate/ glutamine and reduced myo-inositol and choline are hyperammonemia-associated astrocytic changes, while diffusion tensor imaging (DTI) demonstrated changes in neuronal integrity and edema. Linkages among cognition, MRI parameters, and gut microbiota were identified between the groups. Patients with HE had significantly worse cognitive performance, systemic inflammation, dysbiosis, and hyperammonemia than controls and cirrhotic patients without HE. Specific microbial families (autochthonous taxa negatively and Enterobacteriaceae positively) were correlated with MRS and hyperammonemiaassociated astrocytic changes. However, Porphyromonadaceae was only correlated with neuronal changes on DTI without linkages to ammonia. We conclude that specific gut microbial taxa are related to the neuronal and astrocytic consequences of cirrhosis-associated brain dysfunction. Specific microbial families (autochthonous taxa negatively and Enterobacteriaceae positively) were correlated with the MRS and hyperammonemia-associated astrocytic changes (Ahluwalia et al., 2016), which suggests that 


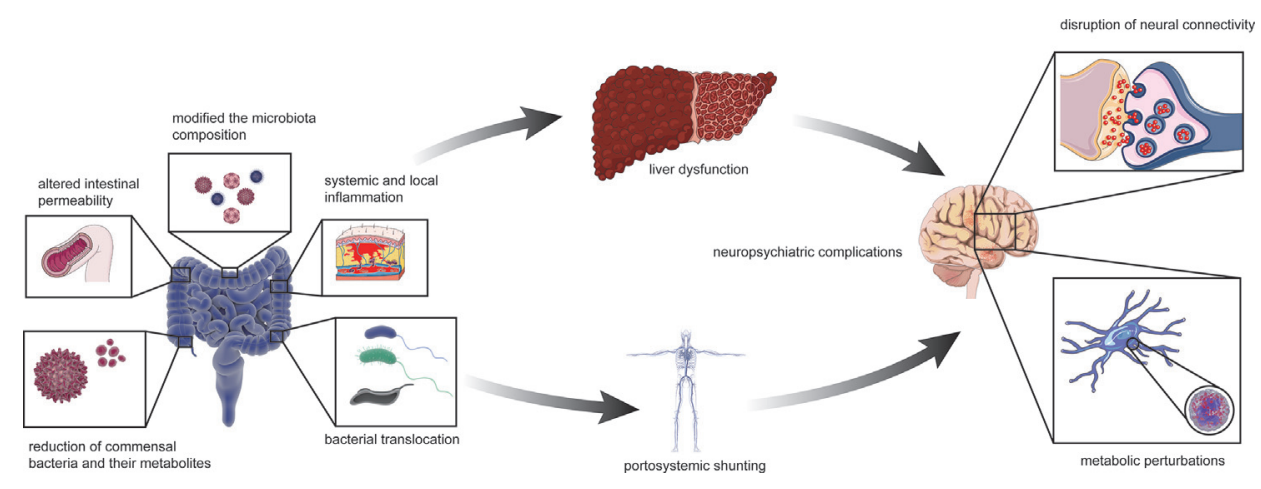

FIGURE 3 | The collapsed gut-liver-brain in HE. The initial factor of gut microbiota dysbiosis can be liver disease possibly with relevant or irrelevant changes of intestinal barrier and portal system. Altered intestinal permeability, bacterial translocation, systemic and local inflammation, modified the microbiota composition, with reduction of commensal bacteria and their metabolites such as short-chain fatty acids, excessive amino and gamma-aminobutyric acid. The abnormal status finally affects liver function such as ammonia metabolism. Besides, liver disease can also cause portal-system shunt and let ammonia escape from the liver. Therefore, ammonia entering the body circulation and then the blood brain barrier, leading to neuropsychiatric complications such as hepatic encephalopathy.

TABLE 1 | Microbiota differences in HE patients' samples

\begin{tabular}{|c|c|c|c|c|c|}
\hline Study & Study design & Samples & Microbiota changes & Potential affection & PMID \\
\hline \multirow[t]{4}{*}{ J. S. Bajaj, et al. } & $\begin{array}{l}\text { Cirrhotics with vs. } \\
\text { without } \mathrm{HE}\end{array}$ & $\begin{array}{l}\text { Mucosal } \\
\text { samples }\end{array}$ & $\begin{array}{l}\text { Enterococcaceae } \uparrow \text { - } \\
\text { Enterococcus } \uparrow\end{array}$ & Intestinal infection and inflammatory response & $\begin{array}{l}21940902 \\
(32)\end{array}$ \\
\hline & & & $\begin{array}{l}\text { Veillonellaceae } \uparrow \text { - } \\
\text { Megasphaera } \uparrow\end{array}$ & N. A & \\
\hline & & & $\begin{array}{l}\text { Bifidobacteriaceae } \uparrow \text { - } \\
\text { Bifidobacterium } \uparrow\end{array}$ & $\begin{array}{l}\text { Regulates synthesis of short-chain fatty acids and } \\
\text { metabolism of oxalate }\end{array}$ & \\
\hline & & & $\begin{array}{l}\text { Lachnospiraceae } \downarrow \text { - } \\
\text { Roseburia } \downarrow\end{array}$ & Regulates bile acid metabolism & \\
\hline \multirow[t]{6}{*}{ Z. Zhang, et al. } & MHE vs. no MHE & Fecal samples & Streptococcus salivarius $\uparrow$ & N. A & $\begin{array}{l}23877352 \\
(33)\end{array}$ \\
\hline & & & Dorea $\uparrow$ & Regulates bile acid metabolism & \\
\hline & & & Subdoligranulum $\uparrow$ & N. A & \\
\hline & & & Incertae sedis XIV $\uparrow$ & N. A & \\
\hline & & & Blautia $\uparrow$ & Lipid metabolism & \\
\hline & & & Roseburia $\uparrow$ & Regulates bile acid metabolism & \\
\hline \multirow[t]{6}{*}{ J. S. Bajaj, et al. } & OHE vs. no OHE & Fecal and & Faecalibacterium $\uparrow$ & Intestinal infection and inflammatory response & 22821944 \\
\hline & & sigmoid & Enterococcus $\downarrow$ & Intestinal infection and inflammatory response & (97) \\
\hline & & mucosal & Burkholderia $\downarrow$ & N. A & \\
\hline & & samples & Proteus $\downarrow$ & Intestinal infection and inflammatory response & \\
\hline & & & Enterobacteriaceae $\uparrow$ & Intestinal infection, inflammatory response and cognitive & \\
\hline & & & Alcaligeneceae $\uparrow$ & inflammatory response and Cognitive & 21940902 \\
\hline \multirow[t]{3}{*}{ J. S. Bajaj, et al. } & OHE vs. no OHE & Fecal samples & Fusobacteriaceae $\uparrow$ & SCFA metabolic & (98) \\
\hline & & & Ruminococcaceae $\downarrow$ & N. A & \\
\hline & & & Lachnospiraceae $\downarrow$ & Intestinal infection and inflammatory response & \\
\hline
\end{tabular}

some specific gut microbial taxa are related to the neuronal and astrocytic consequences of cirrhosis-associated brain dysfunction, indicating important roles in the pathogenesis of HE (Ahluwalia et al., 2016).

\section{CLINICAL AND THERAPEUTIC STRATEGIES}

Based on the comprehensive understanding of pathologic mechanisms and the role of gut microbiota in this process, the clinical implications of the basic research mentioned above are essential to promote the development of therapeutic interventions in HE. There is a growing body of epidemiological and experimental evidence to demonstrate the apparent effect of clinical therapies for HE that act through gut microbiota. Despite these promising results, the side effects and efficacy of therapies for $\mathrm{HE}$ are still being debated. Therefore, comprehensive identification and analysis of multiple treatments that function in modification of gut microbiota are required for further development of gut microbiota strategies for advanced therapies. 


\section{Routine Therapy}

The first serious discussions and analyses of gut microbiotabased therapeutics of HE emerged during the 2010s, and during the past 10 years, much more information has become available for the development of therapeutic strategies. Notably, some of these strategies are even routine treatments in HE, despite the limitations, which will help elucidate the impact of gut microbiota on HE.

\section{Antibiotic Therapy}

Antibiotics usually rapidly sterilize most bacteria. The introduction of antibiotics for non-infectious diseases such as $\mathrm{HE}$ is therefore a feasible option (Ianiro et al., 2016). Rifaximin is a semisynthetic antibiotic designed based on rifamycin, which has been shown to affect cognition in patients with HE by regulating the state of gut microbiota ( $\mathrm{Ng}$ et al., 2019). Increasing evidence shows that rifaximin can significantly increase serum long-chain fatty acid and carbohydrate metabolic intermediate levels in patients with mild HE, which in turn affects serum pro-inflammatory cytokines and secondary bile acids to improve the structure of the gut microbiota and indices of gut immune function (Bajaj, 2016). Research further shows that changes in the metabolism of bacteria-produced agents, such as lipopolysaccharide and deoxycholic acid, caused by rifaximin contribute to maintaining normal gut microbiota levels (DuPont, 2016). In addition, rifaximin treatment significantly reduced the production of gut ammonia via the action of glutamine (Kang et al., 2016). Given the interactions between gut ammonia and gut barrier dysfunction, the detailed and proven molecular mechanisms could be related to gut microbiota (Peleman and Camilleri, 2016). Moreover, rifaximin reduced the diversity and abundance of ammoniaproducing bacteria such as Clostridium and Streptococcus, a risk factor for HE, thus modifying the gut microbiota (Zuo et al., 2017). Notably, although the modulation of the microbiome by rifaximin in patients with $\mathrm{HE}$ was effective, there was no significant change in total gut bacterial load (Kang et al., 2016; Kawaguchi et al., 2019).

\section{Prebiotics Supplementation}

Despite indigestibility by the human, prebiotics have been attracting ever-increasing attention owing to its regulatory effects of microbiota (Swennen et al., 2006). The particularly strong inhibition of intestinal absorption of ammonia in prebiotics was shown in research (Bosques-Padilla and FloresRendón, 2005), as it can cause massive colonic bacteria clearance through the production of hydrogen (Bongaerts et al., 2005). As a kind of prebiotic carbohydrate, lactulose has been applied in clinical practice since 1957 and is currently widely used to treat various intestinal disorders, including HE (Panesar and Kumari, 2011; Nath et al., 2018; Ruszkowski and Witkowski, 2019). The beneficial effects of lactulose may be associated with the effects on microbial metabolism (Tuohy et al., 2002; Ruiz-Aceituno et al., 2020), besides, studies undertaken so far provide conflicting evidence concerning the impact of lactulose on species richness in the gut microbiota (Sarangi et al., 2017; Tetz et al., 2017; Zhai et al., 2018). Therefore, exploring the interaction between lactulose and gut microbiota should be performed. Notably, lactulose can cause reversible qualitative and quantitative changes in fecal flora, which may explain the clinical efficacy of lactulose (Tuohy et al., 2002; Ferreira et al., 2019). A recent randomized controlled trial for patients with HE found showed obvious differences between actinomycetes, Bacteroides, Pachybacteria, and Proteus in the gut of excellent responders and non-responders to lactulose (Wang et al., 2019). This study provides prospective proposals for follow-up studies of lactulose intervention. Besides lactulose, there were other prebiotics to improve brain and liver function in $\mathrm{HE}$ patients. For example, herbal formulations exerted the hepaprotective effect by producing secondary metabolites (Tripathi and Tripathi, 2014). There is also evidence that treatment with fermentable fiber has possible therapeutic benefits in HE patients (Liu et al., 2004).

\section{Probiotic Intervention}

While the effects of intervention are generally positive, antibiotics cannot specifically alter the ecology of the gut microflora (Mahpour et al., 2020). Accompanied by unpredictable adverse effects, these treatments still have certain limitations (de Velde et al., 2018). In contrast, bacteriotherapy has gradually emerged because of its unique advantages. As early as 2011, probiotic interventions were used in bowel diseases such as colitis (Floch et al., 2011). By 2015, evidence from clinical trials showed that the effects of probiotics on gut microbiota have a significant impact on HE (Patel and DuPont, 2015). Further studies indicated that by preventing bacterial adhesion and translocation, probiotics can regulate blood metabolites related to the gut microbiota, such as cytokines, amino acids and vitamins (Qamar, 2015; Usami et al., 2015). Notably, the modification of the gut microflora using probiotics includes changes in the gut microbiota composition. Clostridium and Bifidobacterium were highly enriched, while enterococci and Enterobacteriaceae were significantly reduced, for example (Xia et al., 2018). In addition, probiotics cause changes in the gut microbiota by reducing the number of pathogenic bacteria, ammonia production and absorption as well as endotoxin levels, changing gut mucosal acidification, gut permeability, and the production of short-chain fatty acids, which play an essential role in improving the management of HE (Sharma and Singh, 2016). Recently, a decrease in C-reactive protein $(\mathrm{P}=0.01)$, tumor necrosis factor (TNF) $(\mathrm{P}=0.01)$, FABP-6 $(\mathrm{P}=0.009)$, and claudin-3 $(\mathrm{P}=0.002)$ with a sudden increase in neutrophil oxidation $(\mathrm{P}=0.002)$ was observed in patients with $\mathrm{HE}$ following probiotic intervention (Roman et al., 2019); these factors are associated with enhanced immune adaptations that maintain homeostasis in the gut microbiota (SkoniecznaZydecka et al., 2018). Thus, the results of this study provide further evidence to demonstrate a role for probiotics in the progression of HE. Given the few side effects and the costs of treatment, probiotic intervention has gradually become a common control measure for HE (Rivera-Flores et al., 2019). However, in terms of the degree of intervention, probiotics do not seem superior to lactulose or antibiotics in attaining remission in patients with HE (Cao et al., 2018). Therefore, 
further research on the relationship of prebiotics to gut microflora as well as more efficient treatments is still necessary.

The current medical treatment for $\mathrm{HE}$ is to regulate gut microbiota profiles and reduce ammonia production. Based on this principle and the effects of intervention programs, favorable effects can be observed from using multiple methods to improve HE (Schulz et al., 2019). More than 90\% of patients with HE treated in prospective studies received a combination of rifaximin and lactulose. The clinical application of the combined intervention of probiotics and rifaximin is being actively pursued (Schulz et al., 2016). In addition, it has also been shown that combination of probiotic and prebiotic, also known as synbiotics, can reduce the activity of bacterial urease, which can reduce ammonia production, leading to improvement in HE patient outcomes (Sekhar et al., 2013; Patel and DuPont, 2015). During the past several years, multiple clinical trials and case reports have demonstrated the efficacy of synbiotics play a pivotal role in the treatment of HE. For example, Liu et al. (2004) describe the effect of synbiotic treatment in HE patients (97 study participants). Specifically, after receiving synbiotic treatment, the fecal content of non-urease-producing Lactobacillus species increased significantly, which correlated with a rapid reduction in plasma ammonia levels. However, as mentioned above, all methods have attendant limitations, which have barely been addressed. Therefore, the development of new efficient therapies to directly block or slow disease progression is very important (Weir and Reddy, 2020).

\section{Dietary Intervention}

The composition of the gut microbiota within the human body is strongly associated with dietary habits (Rinninella et al., 2019). Given the important role of gut microbiota in the pathogenesis of $\mathrm{HE}$, dietary change has been recommended as a treatment strategy with the potential to alleviate symptoms in HE (Campion et al., 2019). A recent cross-sectional study in 275 participants shows that lower protein and animal fat intakes in the diets of persons is associated with the increased risk of $\mathrm{HE}$, which is accompanied by differences of gut microbiota, such as Prevotellaceae, Ruminococcaceae and Lachnospiraceae (Bajaj et al., 2020). In addition, it was demonstrated that different kinds of proteins in the diet may contribute towards an improved treatment outcome of $\mathrm{HE}$, branched-chain amino acid intake equally contribute to decrease risk of recurrence in HE (Amodio et al., 2014). Considering the beneficial effects of prebiotics on gut microbiota, it has been convincingly demonstrated that fiber-rich diets can induce beneficial changes on gut microbiota in healthy people, including reduction of Bacteroides spp., Clostridium cluster XIVa bacteria and Enterobacteriaceae, yet their health impact in HE patients is unclear (Merli et al., 2016).

\section{Novel Therapy}

As we are increasingly improving our knowledge about the intestinal microbiota, the limitations of routine therapies in $\mathrm{HE}$, such as the abuse of antibiotics, have led to a dramatic increase in microorganism resistance and have presented increasing challenges to the discovery and development of novel therapies (Laffin et al., 2017).

\section{Fecal Microbiota Transplantation}

Recently, Liu, R. demonstrated that fecal microbial colonization from patients with $\mathrm{HE}$ is associated with neuroinflammation, microglial activation, and ecological disorders by experiments involving colonization in germ-free (GF) mice. This result implied a link among HE, gut microbiota, and fecal microbes (Liu et al., 2020). FMT refers to the transfer of feces from healthy donors to patients with disordered gut microbes. The concept of FMT emerged in the 14th century, but the contribution of FMT to HE has only recently begun to be elucidated (Vindigni and Surawicz, 2017). In 2015, Shen, T. C. et al. first inoculated microbiota in mice with experimental liver injury to reduce the production of ammonia in the gut and the risk of neurotoxicity and encephalopathy associated with hyperammonemia (Shen et al., 2015). Interestingly, a related report suggested the application of FMT in the intervention of HE in that same year (Kang et al., 2015). In 2016, the first case report of FMT for the treatment of HE demonstrated that serial FMT can improve cognitive function in patients with mild HE (Kao et al., 2016). Based on these observations, the first randomized clinical trial in 2017 proved that reasonable donor selection of FMT can reduce the length of hospital stay in patients with HE and improve their cognitive ability and quality of life (Bajaj et al., 2017). Further studies with mouse models of HE indicated that FMT can prevent damage to the gut mucosal immune barrier function and liver necrosis and reduce serum levels of ammonia by enhancing hepatic clearance and gut epithelial permeability (Wang et al., 2017) (Figure 4). The effect of FMT on the gut microbiota is mainly due to the increase in Ruminococcaceae and Bifidobacteriaceae and the decrease in Streptococcaceae and Veillonellaceae. This change was accompanied by an increase in E-cadherin $(P=0.03)$ and defensin alpha $5(P=0.03)$ and a decrease in interleukin-6 $(\mathrm{P}=0.02)$ and serum lipopolysaccharide binding protein (LBP) $(\mathrm{P}=0.009)$ (Bajaj et al., 2019). In 2018, the effect of FMT on regulation of HE was included in the guidelines for use of FMT, increasing the interventions of HE (Mullish et al., 2018). Although the potential of FMT to alter the course of HE is promising, the lack of basic research has led to a lack of understanding about the limitations of FMT (Millan et al., 2017). In addition, infections with microbial pathogens due to lack of donor screening have occurred in multiple retrospective case series (DeFilipp et al., 2019). Therefore, FMT is currently only used in experimental settings and is not a standard treatment.

In addition, potential therapeutic targets in $\mathrm{HE}$ have been discovered, including ammonia scavengers (ornithine phenylacetate) (Alsahhar and Rahimi, 2019), TNP-2092 (a multitargeting drug conjugate with extremely low propensity for resistance development) and gut mannitol (enteral mannitol administration) (Yuan et al., 2020), which are related to gut microbiota. Therefore, further research on the links between gut microbiota and HE may provide prospective guidance for the development of interventions for HE. Based on these results, it is interesting to note that non-pharmacological measures that regulate the gut microbiota, such as exercise and nutritional supplements, may play a major role in modulating the course of HE (Rowland et al., 2018). 


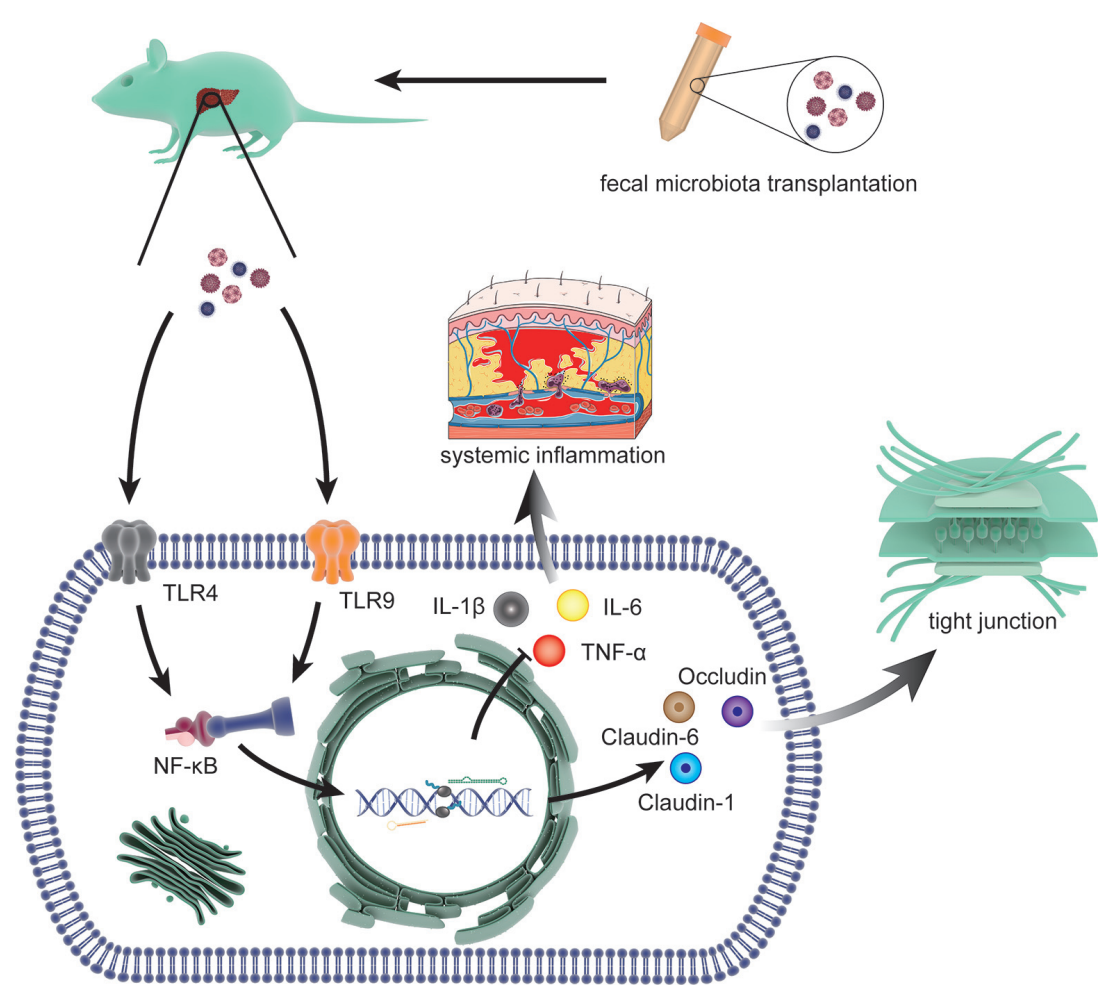

FIGURE 4 | The molecular mechanisms involved in FMT intervention. The process includes limiting the systemic inflammatory response by reducing the expression

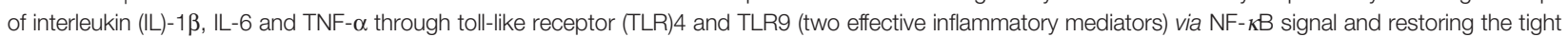
junction proteins (Claudin-1, Claudin-6, and Occludin) that was lost due to HE.

\section{CONCLUSION}

This review aimed to provide the first systematic account of the influence of gut microbiota in hepatic encephalopathy as well as to assess the effects of therapeutic strategies. The findings clearly indicate the regulation of $\mathrm{HE}$ by gut microbiota through a complex signaling network, while some factors emerged as reliable predictors and prognostic biomarkers. The generalizability of this review is mainly limited by its focus solely on cellular and molecular factors; genetic factors were unable to be analyzed, as this subject has not been examined in this field. Notwithstanding these limitations, this work offers valuable insights into the pathogenesis and management strategies of HE from the gut microbiota perspective. Further research on the genetic background of HE related to the gut

\section{REFERENCES}

Aguirre Valadez, J. M., Rivera-Espinosa, L., Mendez-Guerrero, O., ChavezPacheco, J. L., Garcia Juarez, I., and Torre, A. (2016). Intestinal permeability in a patient with liver cirrhosis. Ther. Clin. Risk Manag. 12, 1729-1748. doi: $10.2147 /$ tcrm.S115902

Ahluwalia, V., Betrapally, N. S., Hylemon, P. B., White, M. B., Gillevet, P. M., Unser, A. B., et al. Impaired Gut-Liver-Brain Axis in Patients with Cirrhosis. Sci. Rep. (2016) 6, 1, 1-11. doi: 10.1038/srep26800 microbiota is therefore an essential next step in improving the pathogenic genetic and molecular networks controlling HE.

\section{AUTHOR CONTRIBUTIONS}

All authors listed have made a substantial, direct, and intellectual contribution to the work and approved it for publication.

\section{FUNDING}

This work was supported by funding from the National Natural Science Foundation of China [grant numbers: 81770306, 81971820], the Province Natural Science Foundation of Hunan [grant numbers: 2018JJ2547]. 
Arab, J. P., Martin-Mateos, R. M., and Shah, V. H. (2018). Gut-liver axis, cirrhosis and portal hypertension: the chicken and the egg. Hepatol. Int. 12 (Suppl 1), 24-33. doi: 10.1007/s12072-017-9798-x

Azhari, H., and Swain, M. G. (2018). Role of Peripheral Inflammation in Hepatic Encephalopathy. J. Clin. Exp. Hepatol. 8 (3), 281-285. doi: 10.1016/ j.jceh.2018.06.008

Bajaj, J. S., Ridlon, J. M., Hylemon, P. B., Thacker, L. R., Heuman, D. M., Smith, S., et al. (2012a). Linkage of gut microbiome with cognition in hepatic encephalopathy. Am. J. Physiol. Gastrointest. Liver Physiol. 302 (1), G168G175. doi: 10.1152/ajpgi.00190.2011

Bajaj, J. S., Hylemon, P. B., Ridlon, J. M., Heuman, D. M., Daita, K., White, M. B., et al. (2012b). Colonic mucosal microbiome differs from stool microbiome in cirrhosis and hepatic encephalopathy and is linked to cognition and inflammation. Am. J. Physiol. Gastrointest. Liver Physiol. 303 (6), G675G685. doi: 10.1152/ajpgi.00152.2012

Bajaj, J. S., Kassam, Z., Fagan, A., Gavis, E. A., Liu, E., Cox, I. J., et al. (2017). Fecal microbiota transplant from a rational stool donor improves hepatic encephalopathy: A randomized clinical trial. Hepatology 66 (6), 1727-1738. doi: 10.1002/hep.29306

Bajaj, J. S., Salzman, N. H., Acharya, C., Sterling, R. K., White, M. B., Gavis, E. A., et al. (2019). Fecal Microbial Transplant Capsules Are Safe in Hepatic Encephalopathy: A Phase 1, Randomized, Placebo-Controlled Trial. Hepatology 70 (5), 1690-1703. doi: 10.1002/hep.30690

Bajaj, J. S., Torre, A., Rojas, M. L., Fagan, A., Nandez, I. E., Gavis, E. A., et al. (2020). Cognition and hospitalizations are linked with salivary and faecal microbiota in cirrhosis cohorts from the USA and Mexico. Liver Int. 40 (6), 1395-1407. doi: 10.1111/liv.14437

Bajaj, J. S. (2016). Review article: potential mechanisms of action of rifaximin in the management of hepatic encephalopathy and other complications of cirrhosis. Aliment. Pharmacol. Ther. 43 (Suppl 1), 11-26. doi: 10.1111/ apt.13435

Bajaj, J. S. (2018). Hepatic encephalopathy: classification and treatment. J. Hepatol. 68 (4), 838-839. doi: 10.1016/j.jhep.2017.11.005

Bajaj, J. S. (2019). Gut Microbiota as Biosensors in Patients With Cirrhosis. Cell Mol. Gastroenterol. Hepatol. 8 (2), 231-233. doi: 10.1016/j.jcmgh.2019.04.016

Betrapally, N. S., Gillevet, P. M., and Bajaj, J. S. (2017). Gut microbiome and liver disease. Transl. Res. 179, 49-59. doi: 10.1016/j.trsl.2016.07.005

Biver, E., Berenbaum, F., Valdes, A. M., Araujo de Carvalho, I., Bindels, L. B., Brandi, M. L., et al. (2019). Gut microbiota and osteoarthritis management: An expert consensus of the European society for clinical and economic aspects of osteoporosis, osteoarthritis and musculoskeletal diseases (ESCEO). Ageing Res. Rev. 55, 100946. doi: 10.1016/j.arr.2019.100946

Bongaerts, G., Severijnen, R., and Timmerman, H. (2005). Effect of antibiotics, prebiotics and probiotics in treatment for hepatic encephalopathy. Med. Hypotheses 64 (1), 64-68. doi: 10.1016/j.mehy.2004.07.029

Bosques-Padilla, F. J., and Flores-Rendón, A. R. (2005). [Treatment of hepatic encephalopathy with probiotics and prebiotics]. Rev. Hastroenterol. Mexico 70 (3), 372-374.

Butterworth, R. F. (2019). Hepatic Encephalopathy in Cirrhosis: Pathology and Pathophysiology. Drugs 79 (Suppl 1), 17-21. doi: 10.1007/s40265-018-1017-0

Campion, D., Giovo, I., Ponzo, P., Saracco, G. M., Balzola, F., and Alessandria, C. (2019). Dietary approach and gut microbiota modulation for chronic hepatic encephalopathy in cirrhosis. World J. Hepatol. 11 (6), 489-512. doi: 10.4254/ wjh.v11.i6.489

Cao, Q., Yu, C. B., Yang, S. G., Cao, H. C., Chen, P., Deng, M., et al. (2018). Effect of probiotic treatment on cirrhotic patients with minimal hepatic encephalopathy: A meta-analysis. Hepatobil. Pancreat. Dis. Int. 17 (1), 9-16. doi: 10.1016/j.hbpd.2018.01.005

Covey, A. M., Brody, L. A., Getrajdman, G. I., Sofocleous, C. T., and Brown, K. T. (2004). Incidence, patterns, and clinical relevance of variant portal vein anatomy. AJR Am. J. Roentgenol. 183 (4), 1055-1064. doi: 10.2214/ ajr.183.4.1831055

Dawson, A. M., Mc, L. J., and Sherlock, S. (1957). Neomycin in the treatment of hepatic coma. Lancet (London England) 273 (7008), 1262-1268.

de Velde, F., Mouton, J. W., de Winter, B. C. M., van Gelder, T., and Koch, B. C. P. (2018). Clinical applications of population pharmacokinetic models of antibiotics: Challenges and perspectives. Pharmacol. Res. 134, 280-288. doi: 10.1016/j.phrs.2018.07.005
DeFilipp, Z., Bloom, P. P., Torres Soto, M., Mansour, M. K., Sater, M. R. A., Huntley, M. H., et al. (2019). Drug-Resistant E. coli Bacteremia Transmitted by Fecal Microbiota Transplant. N. Engl. J. Med. 381 (21), 2043-2050. doi: 10.1056/NEJMoa1910437

DuPont, H. L. (2016). Introduction: understanding mechanisms of the actions of rifaximin in selected gastrointestinal diseases. Aliment. Pharmacol. Ther. 43 (Suppl 1), 1-2. doi: 10.1111/apt.13406

Ferreira, M. D. F., Salavati Schmitz, S., Schoenebeck, J. J., Clements, D. N., Campbell, S. M., Gaylor, D. E., et al. (2019). Lactulose drives a reversible reduction and qualitative modulation of the faecal microbiota diversity in healthy dogs. Sci. Rep. 9 (1), 13350. doi: 10.1038/s41598-019-50090-7

Fiuza, C., Salcedo, M., Clemente, G., and Tellado, J. M. (2000). In vivo neutrophil dysfunction in cirrhotic patients with advanced liver disease. J. Infect. Dis. 182 (2), 526-533. doi: 10.1086/315742

Floch, M. H., Walker, W. A., Madsen, K., Sanders, M. E., Macfarlane, G. T., Flint, H. J., et al. (2011). Recommendations for probiotic use-2011 update. J. Clin. Gastroenterol. 45 (Suppl), S168-S171. doi: 10.1097/MCG.0b013e318230928b

Fukui, H., and Wiest, R. (2016). Changes of Intestinal Functions in Liver Cirrhosis. Inflammation Intest. Dis. 1 (1), 24-40. doi: 10.1159/000444436

Hirode, G., Vittinghoff, E., and Wong, R. J. (2019). Increasing Burden of Hepatic Encephalopathy Among Hospitalized Adults: An Analysis of the 2010-2014 National Inpatient Sample. Dig. Dis. Sci. 64 (6), 1448-1457. doi: 10.1007/ s10620-019-05576-9

Ianiro, G., Tilg, H., and Gasbarrini, A. (2016). Antibiotics as deep modulators of gut microbiota: between good and evil. Gut 65 (11), 1906-1915. doi: 10.1136/ gutjnl-2016-312297

Jaffe, A., Lim, J. K., and Jakab, S. S. (2020). Pathophysiology of Hepatic Encephalopathy. Clinics Liver Dis. 24 (2), 175-188. doi: 10.1016/ j.cld.2020.01.002

Jang, J. Y., Kim, G. S., Seo, Y.S., Kim, M. Y., Kim, B. K., Kim, B. S., et al. (2020). KASL clinical practice guidelines for liver cirrhosis: Varices, hepatic encephalopathy, and related complications. Clin. Mol. Hepatol. 26 (2), 83127. doi: $10.3350 / \mathrm{cmh} .2019 .0010 \mathrm{n}$

Kang, D. J., Hylemon, P. B., and Bajaj, J. S. (2015). Fecal transplant to mitigate hyperammonemia and hepatic encephalopathy in animal models. Ann. Hepatol. 14 (5), 762-763. doi: 10.1016/S1665-2681(19)30774-4

Kang, D. J., Kakiyama, G., Betrapally, N. S., Herzog, J., Nittono, H., Hylemon, P. B., et al. (2016). Rifaximin Exerts Beneficial Effects Independent of its Ability to Alter Microbiota Composition. Clin. Transl. Gastroenterol. 7 (8), e187. doi: 10.1038/ ctg.2016.44

Kao, D., Roach, B., Park, H., Hotte, N., Madsen, K., Bain, V., et al. (2016). Fecal microbiota transplantation in the management of hepatic encephalopathy. Hepatology 63 (1), 339-340. doi: 10.1002/hep.28121

Katz, S., Jimenez, M. A., Lehmkuhler, W. E., and Grosfeld, J. L. (1991). Liver bacterial clearance following hepatic artery ligation and portacaval shunt. J. Surg. Res. 51 (3), 267-270. doi: 10.1016/0022-4804(91)90105-u

Kawaguchi, T., Suzuki, F., Imamura, M., Murashima, N., Yanase, M., Mine, T., et al. (2019). Rifaximin-altered gut microbiota components associated with liver/neuropsychological functions in patients with hepatic encephalopathy: An exploratory data analysis of phase II/III clinical trials. Hepatol. Res. 49 (4), 404-418. doi: 10.1111/hepr.13300

Laffin, M., Millan, B., and Madsen, K. L. (2017). Fecal microbial transplantation as a therapeutic option in patients colonized with antibiotic resistant organisms. Gut Microbes 8 (3), 221-224. doi: 10.1080/19490976.2016.1278105

Liu, Q., Duan, Z. P., Ha, D. K., Bengmark, S., Kurtovic, J., and Riordan, S. M. (2004). Synbiotic modulation of gut flora: effect on minimal hepatic encephalopathy in patients with cirrhosis. Hepatology 39 (5), 1441-1449. doi: $10.1002 /$ hep.20194

Liu, R., Kang, J. D., Sartor, R. B., Sikaroodi, M., Fagan, A., Gavis, E. A., et al. (2020). Neuroinflammation in Murine Cirrhosis Is Dependent on the Gut Microbiome and Is Attenuated by Fecal Transplant. Hepatology 71 (2), 611-626. doi: 10.1002/hep.30827

Mahpour, N. Y., Pioppo-Phelan, L., Reja, M., Tawadros, A., and Rustgi, V. K. (2020). Pharmacologic Management of Hepatic Encephalopathy. Clin. Liver Dis. 24 (2), 231-242. doi: 10.1016/j.cld.2020.01.005

Mancini, A., Campagna, F., Amodio, P., and Tuohy, K. M. (2018). Gut : liver : brain axis: the microbial challenge in the hepatic encephalopathy. Food Funct. 9 (3), 1373-1388. doi: 10.1039/c7fo01528c 
Manzhalii, E., Virchenko, O., Falalyeyeva, T., Moiseienko, V., Nykula, T., Kondratiuk, V., et al. (2019). Hepatic Encephalopathy Aggravated by Systemic Inflammation. Dig. Dis. 37 (6), 509-517. doi: 10.1159/000500717

Merli, M., Iebba, V., and Giusto, M. (2016). What is new about diet in hepatic encephalopathy. Metab. Brain Dis. 31 (6), 1289-1294. doi: 10.1007/s11011015-9734-5

Millan, B., Laffin, M., and Madsen, K. (2017). Fecal Microbiota Transplantation: Beyond Clostridium difficile. Curr. Infect. Dis. Rep. 19 (9), 31. doi: 10.1007/ s11908-017-0586-5

Milosevic, I., Vujovic, A., Barac, A., Djelic, M., Korac, M., Radovanovic Spurnic, A., et al. (2019). Gut-Liver Axis, Gut Microbiota, and Its Modulation in the Management of Liver Diseases: A Review of the Literature. Int. J. Mol. Sci. 20 (2), 395. doi: 10.3390/ijms 20020395

Montagnese, S., Russo, F. P., Amodio, P., Burra, P., Gasbarrini, A., Loguercio, C., et al. (2019). Hepatic encephalopathy 2018: A clinical practice guideline by the Italian Association for the Study of the Liver (AISF). Dig. Liver Dis. 51 (2), 190205. doi: 10.1016/j.dld.2018.11.035

Mullish, B. H., Quraishi, M. N., Segal, J. P., McCune, V. L., Baxter, M., Marsden, G. L., et al. (2018). The use of faecal microbiota transplant as treatment for recurrent or refractory Clostridium difficile infection and other potential indications: joint British Society of Gastroenterology (BSG) and Healthcare Infection Society (HIS) guidelines. Gut 67 (11), 1920-1941. doi: 10.1136/ gutjnl-2018-316818

Nath, A., Haktanirlar, G., Varga, Á., Molnár, M. A., Albert, K., Galambos, I., et al. (2018). Biological Activities of Lactose-Derived Prebiotics and Symbiotic with Probiotics on Gastrointestinal System. Med. (Kaunas Lithuania) 54 (2), 18. doi: 10.3390/medicina54020018

Ng, K. M., Aranda-Díaz, A., Tropini, C., Frankel, M. R., Van Treuren, W., O'Laughlin, C. T., et al. (2019). Recovery of the Gut Microbiota after Antibiotics Depends on Host Diet, Community Context, and Environmental Reservoirs. Cell Host Microbe 26 (5), 650-65.e4. doi: 10.1016/ j.chom.2019.10.011

Panesar, P. S., and Kumari, S. (2011). Lactulose: production, purification and potential applications. Biotechnol. Adv. 29 (6), 940-948. doi: 10.1016/ j.biotechadv.2011.08.008

Patel, R., and DuPont, H. L. (2015). New approaches for bacteriotherapy: prebiotics, new-generation probiotics, and synbiotics. Clin. Infect. Dis. 60 (Suppl 2), S108-S121. doi: 10.1093/cid/civ177

Patidar, K. R., and Bajaj, J. S. (2015). Covert and Overt Hepatic Encephalopathy: Diagnosis and Management. Clin. Gastroenterol. Hepatol. Off. Clin. Pract. J. Am. Gastroenterol. Assoc. 13 (12), 2048-2061. doi: 10.1016/ j.cgh.2015.06.039

Peleman, C., and Camilleri, M. (2016). Rifaximin, Microbiota Biology, and Hepatic Encephalopathy. Clin. Transl. Gastroenterol. 7 (10), e195. doi: $10.1038 /$ ctg.2016.52

Qamar, A. A. (2015). Probiotics in Nonalcoholic Fatty Liver Disease, Nonalcoholic Steatohepatitis, and Cirrhosis. J. Clin. Gastroenterol. 49 (Suppl 1), S28-S32. doi: $10.1097 / \mathrm{mcg} .0000000000000347$

Raj, D., Tomar, B., Lahiri, A., and Mulay, S. R. (2020). The gut-liver-kidney axis: Novel regulator of fatty liver associated chronic kidney disease. Pharmacol. Res. 152, 104617. doi: 10.1016/j.phrs.2019.104617

Rinninella, E., Raoul, P., Cintoni, M., Franceschi, F., Miggiano, G. A. D., Gasbarrini, A., et al. (2019). What is the Healthy Gut Microbiota Composition? A Changing Ecosystem across Age, Environment, Diet, and Diseases. Microorganisms 7 (1), 14. doi: 10.3390/microorganisms7010014

Rivera-Flores, R., Moran-Villota, S., Cervantes-Barragan, L., Lopez-Macias, C., and Uribe, M. (2019). Manipulation of microbiota with probiotics as an alternative for treatment of hepatic encephalopathy. Nutrition 73, 110693. doi: 10.1016/j.nut.2019.110693

Rodrigo, R., Cauli, O., Gomez-Pinedo, U., Agusti, A., Hernandez-Rabaza, V., Garcia-Verdugo, J. M., et al. (2010). Hyperammonemia induces neuroinflammation that contributes to cognitive impairment in rats with hepatic encephalopathy. Gastroenterology 139 (2), 675-684. doi: 10.1053/ j.gastro.2010.03.040

Roman, E., Nieto, J. C., Gely, C., Vidal, S., Pozuelo, M., Poca, M., et al. (2019). Effect of a Multistrain Probiotic on Cognitive Function and Risk of Falls in Patients With Cirrhosis: A Randomized Trial. Hepatol. Commun. 3 (5), 632645. doi: $10.1002 /$ hep 4.1325
Rowland, I., Gibson, G., Heinken, A., Scott, K., Swann, J., Thiele, I., et al. (2018). Gut microbiota functions: metabolism of nutrients and other food components. Eur. J. Nutr. 57 (1), 1-24. doi: 10.1007/s00394-017-1445-8

Ruiz-Aceituno, L., Esteban-Torres, M., James, K., Moreno, F. J., and van Sinderen, D. (2020). Metabolism of biosynthetic oligosaccharides by human-derived Bifidobacterium breve UCC2003 and Bifidobacterium longum NCIMB 8809. Int. J. Food Microbiol. 316, 108476. doi: 10.1016/j.ijfoodmicro.2019.108476

Ruszkowski, J., and Witkowski, J. M. (2019). Lactulose: Patient- and dosedependent prebiotic properties in humans. Anaerobe 59, 100-106. doi: 10.1016/j.anaerobe.2019.06.002

Sarangi, A. N., Goel, A., Singh, A., Sasi, A., and Aggarwal, R. (2017). Faecal bacterial microbiota in patients with cirrhosis and the effect of lactulose administration. BMC Gastroenterol. 17 (1), 125. doi: 10.1186/s12876-0170683-9

Schnabl, B., and Brenner, D. A. (2014). Interactions between the intestinal microbiome and liver diseases. Gastroenterology 146 (6), 1513-1524. doi: 10.1053/j.gastro.2014.01.020

Schulz, C., Schutte, K., Kropf, S., Schmitt, F. C., Vasapolli, R., Kliegis, L. M., et al. (2016). RiMINI - the influence of rifaximin on minimal hepatic encephalopathy (MHE) and on the intestinal microbiome in patients with liver cirrhosis: study protocol for a randomized controlled trial. Trials 17 (1), 111. doi: 10.1186/s13063-016-1205-8

Schulz, C., Schutte, K., Vilchez-Vargas, R., Vasapolli, R., and Malfertheiner, P. (2019). Long-Term Effect of Rifaximin with and without Lactulose on the Active Bacterial Assemblages in the Proximal Small Bowel and Faeces in Patients with Minimal Hepatic Encephalopathy. Dig. Dis. 37 (2), 161-169. doi: $10.1159 / 000494216$

Sekhar, M. S., Unnikrishnan, M. K., Rodrigues, G. S., and Mukhopadhyay, C. (2013). Synbiotic formulation of probiotic and lactulose combination for hepatic encephalopathy treatment: a realistic hope? Med. Hypotheses 81 (2), 167-168. doi: 10.1016/j.mehy.2013.05.016

Sharma, B. C., and Singh, J. (2016). Probiotics in management of hepatic encephalopathy. Metab. Brain Dis. 31 (6), 1295-1301. doi: 10.1007/s11011016-9826-x

Shen, T. C., Albenberg, L., Bittinger, K., Chehoud, C., Chen, Y. Y., Judge, C. A., et al. (2015). Engineering the gut microbiota to treat hyperammonemia. J. Clin. Invest. 125 (7), 2841-2850. doi: 10.1172/jci79214

Sipeki, N., Antal-Szalmas, P., Lakatos, P. L., and Papp, M. (2014). Immune dysfunction in cirrhosis. World J. Gastroenterol. 20 (10), 2564-2577. doi: 10.3748/wjg.v20.i10.2564

Skonieczna-Zydecka, K., Kaczmarczyk, M., Loniewski, I., Lara, L. F., Koulaouzidis, A., Misera, A., et al. (2018). A Systematic Review, Meta-Analysis, and Meta-Regression Evaluating the Efficacy and Mechanisms of Action of Probiotics and Synbiotics in the Prevention of Surgical Site Infections and Surgery-Related Complications. J. Clin. Med. 7 (12), 556. doi: 10.3390/jcm7120556

Summerskill, W. H. (1958). Hepatic coma in liver failure and gastro-intestinal haemorrhage treated with neomycin. Br. Med. J. 2 (5108), 1322-1325. doi: $10.1136 / \mathrm{bmj} .2 .5108 .1322$

Swennen, K., Courtin, C. M., and Delcour, J. A. (2006). Non-digestible oligosaccharides with prebiotic properties. Crit. Rev. Food Sci. Nutr. 46 (6), 459-471. doi: 10.1080/10408390500215746

Tetz, G. V., Ruggles, K. V., Zhou, H., Heguy, A., Tsirigos, A., and Tetz, V. (2017). Bacteriophages as potential new mammalian pathogens. Sci. Rep. 7 (1), 7043. doi: 10.1038/s41598-017-07278-6

Tripathi, S., and Tripathi, Y. B. (2014). Hepatic encephalopathy: cause and possible management with botanicals. Recent Patents Inflammation Allergy Drug Discovery 8 (3), 185-191. doi: 10.2174/1872213x08666141107164418

Tuohy, K. M., Ziemer, C. J., Klinder, A., Knoebel, Y., Pool-Zobel, B. L., and Gibson, G. R. (2002). A human volunteer study to determine the prebiotic effects of lactulose powder on human colonic microbiota. Microb. Ecol. Health Dis. 14 (3), 165-173. doi: 10.1080/089106002320644357

Usami, M., Miyoshi, M., and Yamashita, H. (2015). Gut microbiota and host metabolism in liver cirrhosis. World J. Gastroenterol. 21 (41), 11597-11608. doi: 10.3748/wjg.v21.i41.11597

Vindigni, S. M., and Surawicz, C. M. (2017). Fecal Microbiota Transplantation. Gastroenterol. Clin. North Am. 46 (1), 171-185. doi: 10.1016/j.gtc.2016.09.012

Wang, W. W., Zhang, Y., Huang, X. B., You, N., Zheng, L., and Li, J. (2017). Fecal microbiota transplantation prevents hepatic encephalopathy in rats with 
carbon tetrachloride-induced acute hepatic dysfunction. World J. Gastroenterol. 23 (38), 6983-6994. doi: 10.3748/wjg.v23.i38.6983

Wang, J. Y., Bajaj, J. S., Wang, J. B., Shang, J., Zhou, X. M., Guo, X. L., et al. (2019). Lactulose improves cognition, quality of life, and gut microbiota in minimal hepatic encephalopathy: A multicenter, randomized controlled trial. J. Dig. Dis. 20 (10), 547-556. doi: 10.1111/1751-2980.12816

Weir, V., and Reddy, K. R. (2020). Nonpharmacologic Management of Hepatic Encephalopathy: An Update. Clinics Liver Dis. 24 (2), 243-261. doi: 10.1016/ j.cld.2020.01.003

Wiest, R., Albillos, A., Trauner, M., Bajaj, J. S., and Jalan, R. (2017). Targeting the gut-liver axis in liver disease. J. Hepatol. 67 (5), 1084-1103. doi: 10.1016/ j.jhep.2017.05.007

Xia, X., Chen, J., Xia, J., Wang, B., Liu, H., Yang, L., et al. (2018). Role of probiotics in the treatment of minimal hepatic encephalopathy in patients with HBVinduced liver cirrhosis. J. Int. Med. Res. 46 (9), 3596-3604. doi: 10.1177/ 0300060518776064

Xu, L., Zheng, X., Wang, Y., Fan, Q., Zhang, M., Li, R., et al. (2018). Berberine protects acute liver failure in mice through inhibiting inflammation and mitochondria-dependent apoptosis. Eur. J. Pharmacol. 819, 161-168. doi: 10.1016/j.ejphar.2017.11.013

Xu, X. Y., Ding, H. G., Li, W. G., Jia, J. D., Wei, L., Duan, Z. P., et al. (2019). Chinese guidelines on management of hepatic encephalopathy in cirrhosis. World J. Gastroenterol. 25 (36), 5403-5422. doi: 10.3748/wjg.v25.i36.5403

Yanny, B., Winters, A., Boutros, S., and Saab, S. (2019). Hepatic Encephalopathy Challenges, Burden, and Diagnostic and Therapeutic Approach. Clin. Liver Dis. 23 (4), 607-623. doi: 10.1016/j.cld.2019.07.001
Yuan, Y., Wang, X., Xu, X., Liu, Y., Li, C., Yang, M., et al. (2020). Evaluation of a Dual-Acting Antibacterial Agent, TNP-2092, on Gut Microbiota and Potential Application in the Treatment of Gastrointestinal and Liver Disorders. ACS Infect. Dis. 6 (5), 820-831. doi: 10.1021/acsinfecdis.9b00374

Zhai, S., Zhu, L., Qin, S., and Li, L. (2018). Effect of lactulose intervention on gut microbiota and short chain fatty acid composition of C57BL/6J mice. Microbiologyopen 7 (6), e00612. doi: 10.1002/mbo3.612

Zhang, Z., Zhai, H., Geng, J., Yu, R., Ren, H., Fan, H., et al. (2013). Large-scale survey of gut microbiota associated with MHE Via 16S rRNA-based pyrosequencing. Am. J. Gastroenterol. 108 (10), 1601-1611. doi: 10.1038/ ajg.2013.221

Zuo, Z., Fan, H., Tang, X. D., Chen, Y. M., Xun, L. T., Li, Y., et al. (2017). Effect of different treatments and alcohol addiction on gut microbiota in minimal hepatic encephalopathy patients. Exp. Ther. Med. 14 (5), 4887-4895. doi: 10.3892/etm.2017.5141

Conflict of Interest: The authors declare that the research was conducted in the absence of any commercial or financial relationships that could be construed as a potential conflict of interest.

Copyright $\odot 2021 \mathrm{Liu}, \mathrm{Xu}$ and Jiang. This is an open-access article distributed under the terms of the Creative Commons Attribution License (CC BY). The use, distribution or reproduction in other forums is permitted, provided the original author(s) and the copyright owner(s) are credited and that the original publication in this journal is cited, in accordance with accepted academic practice. No use, distribution or reproduction is permitted which does not comply with these terms. 